\title{
Compensatory growth of lateral roots responding to excision of seminal root tip in rice
}

\author{
Tsubasa Kawai ${ }^{1}$, Misuzu Nosaka-Takahashi ${ }^{2}$, Akira Yamauchi ${ }^{1}$, Yoshiaki Inukai ${ }^{2,3}$ \\ ${ }^{1}$ Graduate School of Bioagricultural Sciences, Nagoya University, Nagoya, Aichi 464-8601, Japan \\ ${ }^{2}$ International Cooperation Center for Agricultural Education, Nagoya University, Nagoya, Aichi 464-8601, Japan \\ ${ }^{3}$ PREST, JST, Kawaguchi, Saitama 332-0012, Japan \\ Corresponding author: Y. Inukai, E-mail: inukaiy@agr.nagoya-u.ac.jp, Phone: +81-52-789-4073, Fax: +81-52-789- \\ 4222
}

Received on January 30, 2017; Accepted on May 11, 2017

\begin{abstract}
Development of lateral roots (LRs) is promoted when parental root elongation is suppressed. Promotion of LR growth compensates for parental root growth, which contributes to the maintenance of total root length. However, the developmental processes underlying the compensatory growth of LRs are still unclear. In this study, we induced LR compensatory growth in rice by surgical excision of a parent (seminal) root tip, and analyzed the morphological and anatomical changes in LRs. Our analysis revealed that seminal roottip excision increased the diameter of first-order LRs by increasing in the number of ground-tissue layers and the stele diameter and also promoted elongation, with higher order LRs emerging in the remaining proximal portions. We also found that LR primordia in early developmental stages increased in diameter markedly and then produced higher order LRs pronouncedly in response to seminal-root cutting. Furthermore, the degree of change in LR morphology differed depending on the distance from the cut site, with changes in morphologies weakening further from the cut site. Taken together, the morphological and anatomical changes of LRs that are induced by the root-tip excision of the parent vary continuously, and are dependent on both the developmental stage of LR primordia at the site of root-cutting and the distance from the cut site.
\end{abstract}

Keywords: compensatory growth, lateral root, lateral root primordia, rice (Oryza sativa L.), root tip excision

Abbreviations: CRs, crown roots; LR, lateral root; LRP, lateral root primordia; SR, seminal root

\section{Introduction}

The root systems of rice are composed of several different types of roots. A seminal root is produced embryonically, whereas crown roots develop postembryonically from the stem. Seminal and crown roots (parent roots) produce lateral roots (LRs), which are classified into S-types and L-types according to distinct morphological and anatomical characteristics (Kawata and Shibayama 1965, Kono et al. 1972, Yamauchi et al. 1987); S-type LRs are short and thin, and never produce higher order LRs, whereas L-type LRs are long and thick, and often produce higher order LRs. L-type LRs are more similar anatomically to parent roots - for example in developed vascular structure, and the presence of sclerenchyma and multiple cortex layers - than are S-types. These two different types of LRs vary in developmental characteristics and genetic control of their development (Kawata and Shibayama 1965, Sasaki et al. 1984, Wang et al. 2006, Niones et al. 2015). In addition, production of L-type LRs is important for expanding the root system because of their ability to produce higher order LRs.

Development of LRs is promoted when elongation of the parent root is inhibited; one such factor inhibiting parent root elongation is soil compaction, as compact soils, which are heterogeneously distributed, causes mechanical impedance of parent root growth. This mechanical impedance of the parent root triggers LR development in various plant species (Goss 1977, Shierlaw and Alston 1984, Atwell 1990, Montagu et al. 2001). Even in compact soil, LRs can elongate into the narrow pore spaces and cracks in the soil (Bingham and Bengough 2003, White and

Kawai T, Nosaka-Takahashi M, Yamauchi A, Inukai Y 2017 Compensatory growth of lateral roots responding to excision of seminal root tip in rice. Plant Root 11:48-57. doi:10.3117/plantroot.11.48

Copyrights 2017, Plant Root (JSRR), www.plantroot.org 
Kirkegaard 2010, Haling et al. 2011). Compensatory growth of LRs facilitates the uptake of water and nutrients by maintaining sufficient total root length, which in turn contributes to shoot growth maintenance (Montagu et al. 2001). However, the developmental processes underlying compensatory LR growth remain largely unknown.

Compensatory growth of LRs can be also induced by surgical excision of the root tip, which disrupts parent root elongation and stimulates the development of L-type LRs in the remaining proximal portions of the root (Sasaki et al. 1984). The ability of a plant to produce higher order LRs in response to the root-tip excision is defined by the developmental stage of the lateral root primordia (LRP); however, morphological and anatomical changes induced by the root-tip excision and their relation with the developmental stage of LRP have yet to be determined.

Here, we examined the morphological and anatomical changes in LRs induced by seminal-root cutting, focusing on the developmental stage of LRP at the location of the root cut and the LR position relative to the cut site. Morphological changes in LRs induced by seminal-root cutting varied continuously according to the developmental stage of LRP at the root-cutting site and the LR position relative to the cut site. We also found that the anatomical characteristics of LRs were different depending on the developmental stage of LRP at the root-cutting site. Moreover, analyses of the relationships between several LR characteristics revealed that larger diameter LRs grow longer and produce second-order LRs more frequently than do smaller diameter LRs.

\section{Materials and Methods}

\section{Plant growth conditions and root-cutting treatment}

Seedlings of rice (Oryza sativa L. var. Taichung 65) were used in all experiments. Rice seeds were sterilized by soaking in a Benlate solution dissolved in tap water $\left(2.5 \mathrm{~g} \mathrm{~L}^{-1}\right)$ at $28^{\circ} \mathrm{C}$ for $3 \mathrm{~d}$, then rinsed three times with tap water and transferred onto plastic nets floating on nutrient-free filtered water in a black plastic box. The water was aerated with a pump (OX-30, Tetra-Japan, Japan) beginning $2 \mathrm{~d}$ after seedling transference and kept at a constant level through the addition of filtered water. Seedlings were grown in a growth chamber (MLR351 , Sanyo, Japan) at $28^{\circ} \mathrm{C}$ under continuous light. Seminal root length and the distance from the base to the LR emergence site, where the youngest LR appears from the outer surface of the seminal root, were measured $4 \mathrm{~d}$ after transference (Fig. 1A).
Seedlings having more than $10 \mathrm{~mm}$ distance from the base to the LR emergence site as like Fig. 3A were used for the following experiments. The seminal roots were cut into various root-cutting ratios using a cutting knife (Fig. 2A). Root-cutting ratio $(\%)$ was calculated as:

$$
\frac{\text { Distance behind root tip }(\mathrm{mm})}{\text { Distance from root tip to LR emergence site }(\mathrm{mm})} \times 100
$$

After root cutting, seedlings were put back onto the floating nets and allowed to continue to grow.

\section{Morphological and anatomical characterization}

Measurement of emerged LR characteristics: Seminal roots were sampled and fixed in FAA (5\% formaldehyde, $5 \%$ acetic acid, $63 \%$ ethanol $\left[\mathrm{v} \mathrm{v}^{-1}\right]$ ) $10 \mathrm{~d}$ following root cutting (14 $\mathrm{d}$ after transplanting). Diameters of first-order LRs were measured at the basal region using a microscope (IX71, Olympus, Japan) and a micrometer. For the measurement of LR length, LRs, along with the seminal root, were fully extended on an agar plate, and images were taken with a digital camera (D90, Nikon, Japan) at 300 dpi. Length of the first-order LR was measured using image-analysis software (LIA for Win32, https:// www.agr.nagoya-u.ac.jp/ shinkan/LIA32). For evaluating the effect of root cutting in the different root-cutting ratios, morphology of the LRs that emerged in the region between the excision site and a $10 \%$ higher ratio were analyzed (Fig. 3A). For analyzing the relationship between morphological changes in LR induced by the root-tip excision and distance from the cut site to each LR, morphology of the LRs that emerged in the $10 \%$ root-cutting ratio were analyzed every $5 \mathrm{~mm}$ up to a distance of $25 \mathrm{~mm}$ from the cut site (Fig. 5A).

Observation of LRP by rapid transparency method: Seminal roots of 4-d-old seedlings were fixed and cleared with TOMEI-I (Hasegawa et al. 2016). Images of LRP in the cleared seminal roots were captured using a differential interference microscope (BX60, Olympus, Japan), and distance from root tip to the LR emergence site and distance from root tip to the LRP were measured. LRP position was defined as relative distance from root tip, which is distance from root tip to LRP based on distance from root tip to the LR emergence site as 100\% (Fig. 1A). Width of the meristem region, excluding the 'Tasche' (which originates from the endodermis of the parent root; Kawata and Shibayama, 1965) and distance from the base to the tip were measured as LRP diameter and length using imaging software (LIA for Win32), respectively (Fig. 1B). 
Histological analysis of LRs: The thickest LR among the LRs that emerged $4 \mathrm{~d}$ following $10 \%$, $50 \%, 60 \%, 80 \%$ and $100 \%$ root-cutting in each plant were sampled. The sampled LRs were placed in FAA solution, and de-aerated with a vacuum for 30 min. The FAA solution was then renewed and samples were kept for $24 \mathrm{~h}$ at $4^{\circ} \mathrm{C}$. Fixed samples were dehydrated in a graded ethanol series and embedded in Technovit 7100 (Heraeus Kulzer, Germany); embedded samples were then sectioned into $6-\mu \mathrm{m}$-thick slices using a rotary microtome (RM2125 RTS, Leica, Germany) and observed under a microscope (IX71, Olympus, Japan). Stele diameters and thicknesses of the ground tissues (exodermis, sclerenchyma, cortex, and endodermis) were measured using imaging software (LIA for Win32), and the number of ground-tissue layers were counted (Fig. 4A).

\section{Data analysis}

The morphological and anatomical characteristics of the LRs of the different groups were compared using ANOVA and a multiple comparison Tukey's test in R commander ver. 2.3-1 (Fox 2005). The relationships between relative distance from root tip and LRP size was analyzed using regression analysis, and correlation coefficients were calculated in Microsoft Excel 2016.

\section{Results}

Relationship between developmental stage of LRP and responsiveness to root-tip excision

For determining the relationship between LRP developmental stage and responsiveness to the roottip excision, we first analyzed the LRP developmental stage in the intact seminal roots of 4-d-old seedlings. As a result, positive correlations were detected between LRP size (diameter or length) and relative distance from root tip, which were stronger than the correlations between LRP size (diameter or length) and the distance from root tip to LRP ( $r=0.68$ and 0.64 respectively; data not shown), suggesting that LRP located at the same relative distance from root tip within root tip to the LR emergence site are at the same developmental stages in the seminal roots of 4-d-old seedlings (Fig. 1A-D; see Materials and Methods).

Based on the revealed relationship between LRP developmental stage and relative distance from root

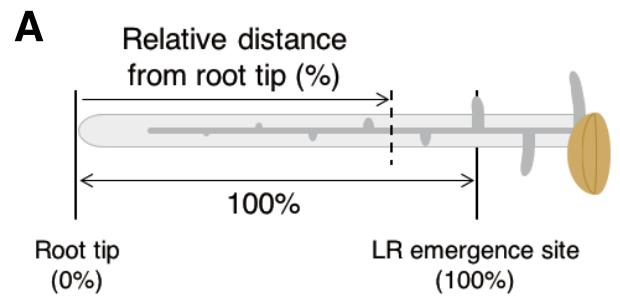

B
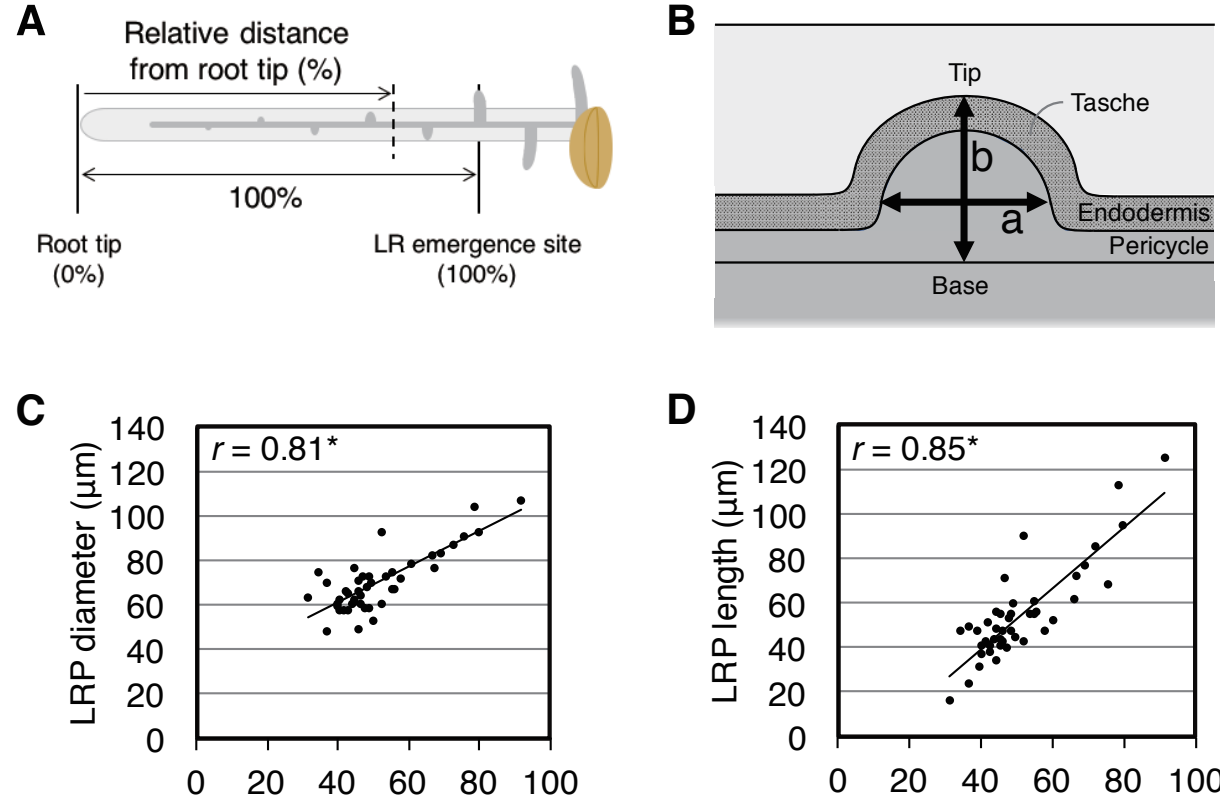

D

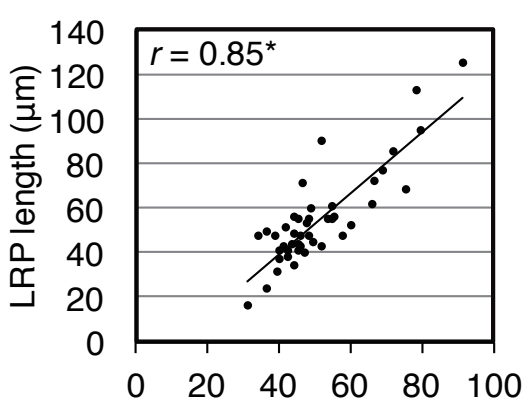

Relative distance from root tip (\%)

Fig. 1. Relationship between position and size of LRP on intact seminal roots. (A) Schematic diagram of a 4-d-old seminal root. LRP position was defined as the relative distance from root tip based on distance from root tip to the LR emergence site, where the youngest LR appears from the outer surface of the seminal root, as $100 \%$. (B) Schematic diagram of LRP in a seminal root; LRP diameter and length were defined as the width of the meristem region (a) and the distance from the base to tip (b), respectively. (C, D) Relationships between relative distance from root tip to primordia within root tip to the LR emergence site and diameter (C) or length (D) of LRP in seminal roots. Asterisk indicates a significant correlation between relative distance from root tip and LRP diameter or length $(P<0.01, \mathrm{n}=44$ primordia from 5 plants $)$. 
tip, seminal roots were cut into various root-cutting ratios for determining the relationship between LRP developmental stage and responsiveness to the roottip excision (Fig. 2A; see Materials and Methods). No LRs with higher order LRs emerged from intact seminal roots. For root-cutting ratios of less than $60 \%$, maximum diameter of first-order LR (hereinafter diameter of first-order LR is referred to simply as LR diameter), which is a diameter of the thickest LR among the LRs that emerged after root cutting, was greater than $160 \mu \mathrm{m}$ (control with intact roots: $104.0 \pm 4.6 \mu \mathrm{m}, \mathrm{n}=30$ plants), and almost all of the thickest LRs produced second-order LRs (Fig. 2B). For root-cutting ratios of more than $60 \%$, increases in the maximum LR diameter gradually slowed along with increasing root-cutting ratio, and few LRs produced second-order LRs.

For a more quantitative evaluation of the relationship between LRP developmental stage and responsiveness to the root-tip excision, seminal roots were cut into $10 \%, 50 \%, 60 \%, 80 \%$, and $100 \%$ root-cutting ratios, and morphology of the LRs emerged after root cutting were analyzed (Fig. 3A; see Materials and Methods). Maximum LR diameters were virtually identical in the $10 \%$ and $50 \%$ root-cutting ratios, and LRs with second-order
LRs emerged equally in both (Fig. 3B, C, G, H). In the $60 \%$ root-cutting ratio, the increase in the maximum LR diameter induced by root cutting was smaller than those of the $10 \%$ and $50 \%$ root-cutting ratios, and few LRs with second-order LRs emerged (Fig. 3D, G, H). The maximum diameter of LRs was slightly higher in the $80 \%$ root-cutting ratio than that of control (Fig. 3E, G). Notably, some LRs with second-order LRs that sometimes emerged in the $80 \%$ root-cutting ratio had a much smaller diameter (about $120 \mu \mathrm{m}$ ) than those that emerged in the $10 \%$ and $50 \%$ root-cutting ratios. In the $100 \%$ rootcutting ratio, LR diameter was unaffected by root cutting, and no LRs produced higher order LRs (Fig. 3F, G, H). The diameter of LRs that emerged in the region between the excision site and a 10\% higher ratio was distributed successively in the $10 \%, 50 \%$, $60 \%$, and $80 \%$ root-cutting ratios (Fig. 3I), and LRs with second-order LRs were more often of a larger diameter in each root-cutting ratio.

Anatomical changes of LR induced by seminal-root cutting in the different developmental stages of LRP

We analyzed the anatomical characteristics of LRs of various diameters emerged in the different root-

\section{A}

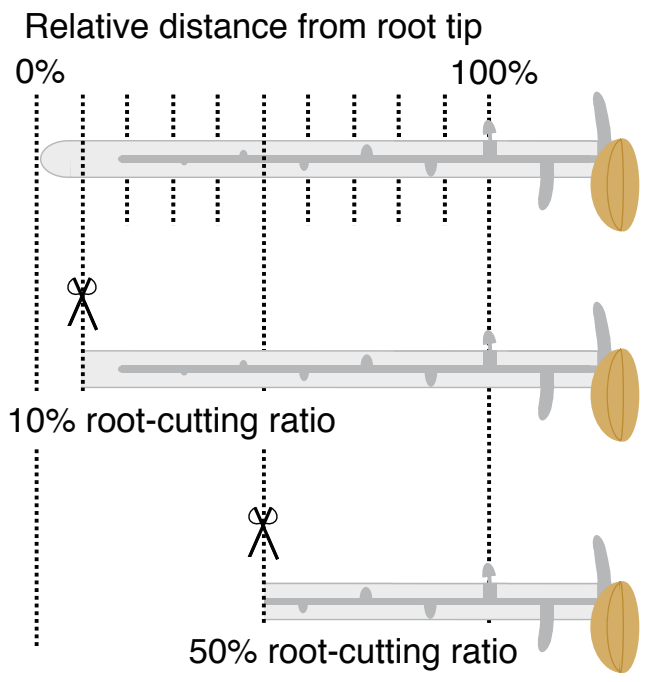

B

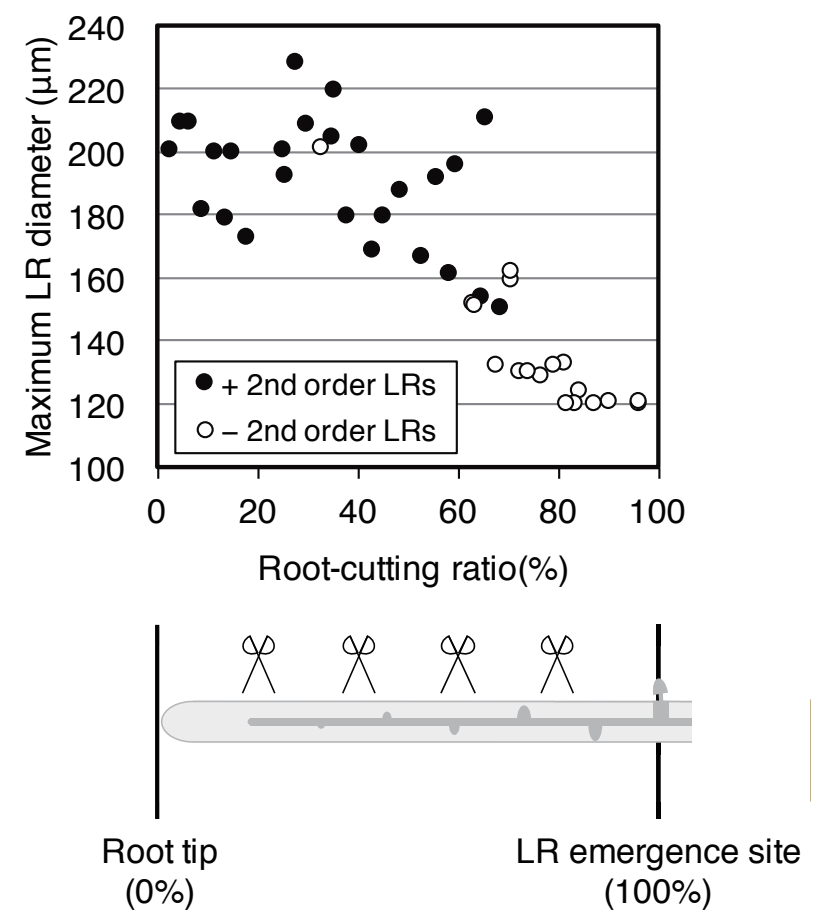

Fig. 2. Relationship between root-cutting ratio and LR morphology. (A) Schematic diagram showing the definition of the rootcutting ratio. (B) Seminal roots were cut into various root-cutting ratios. Diameter of the thickest LR among the LRs that emerged after root cutting in each plant and whether second-order LRs were produced are shown ( $\mathrm{n}=44$ plants). 

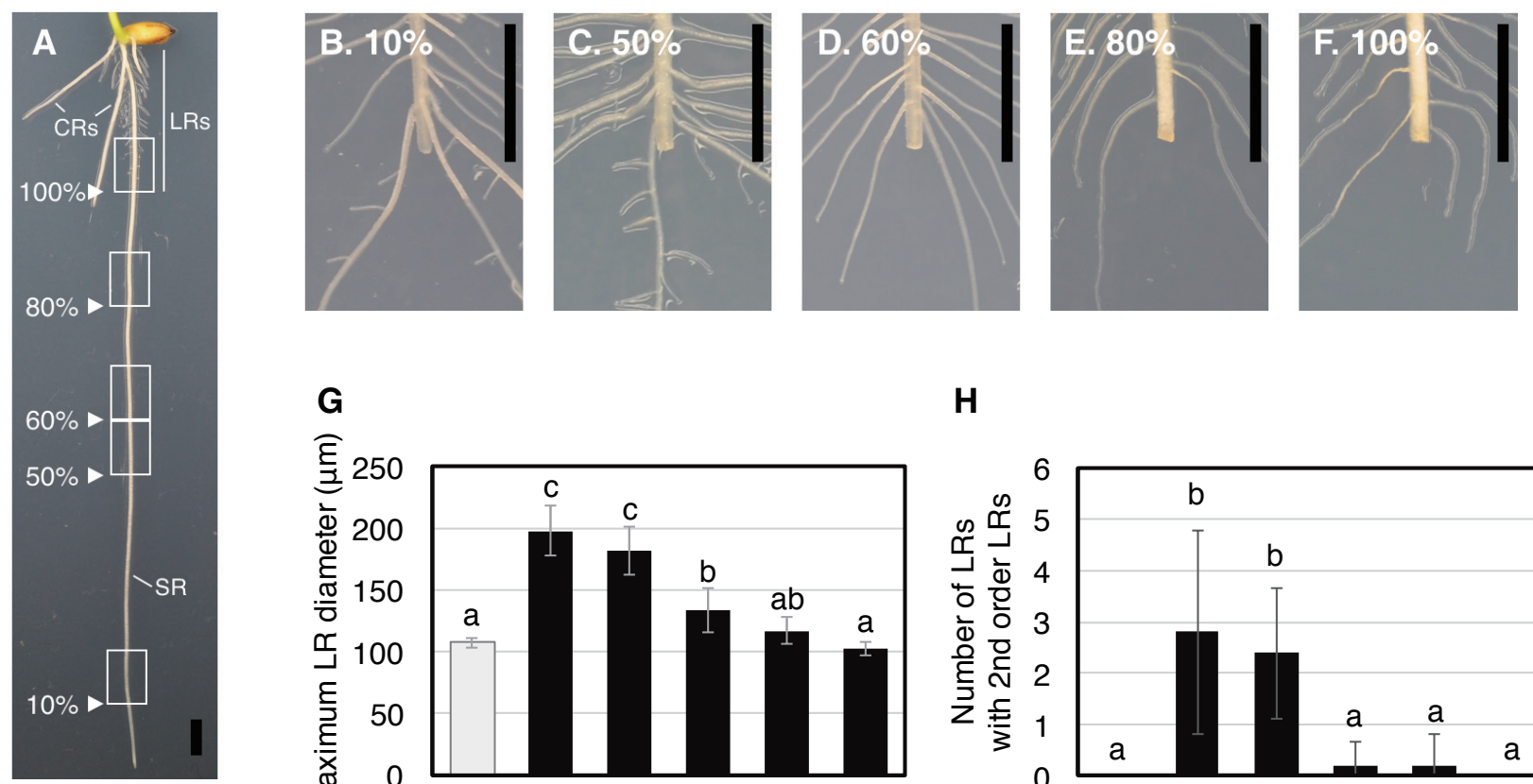

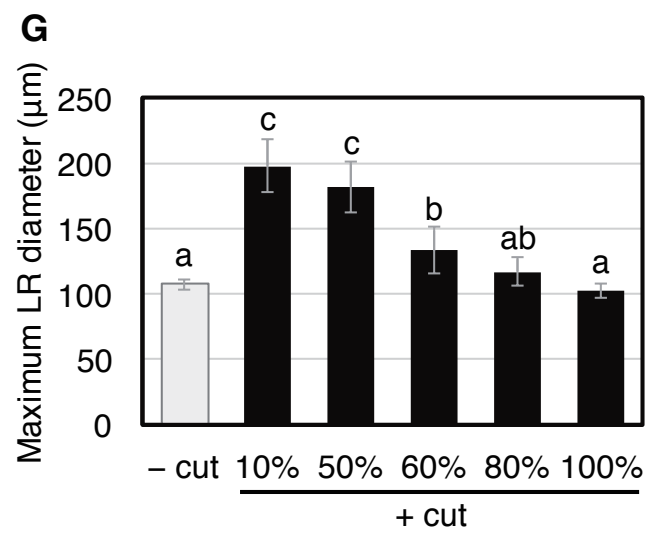

Root-cutting ratio
H

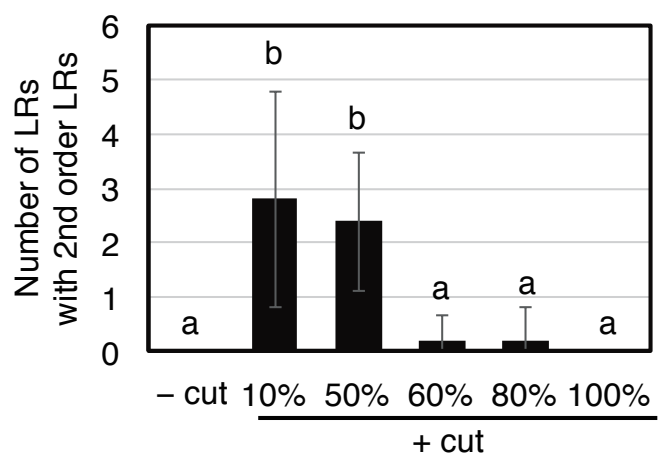

Root-cutting ratio

I

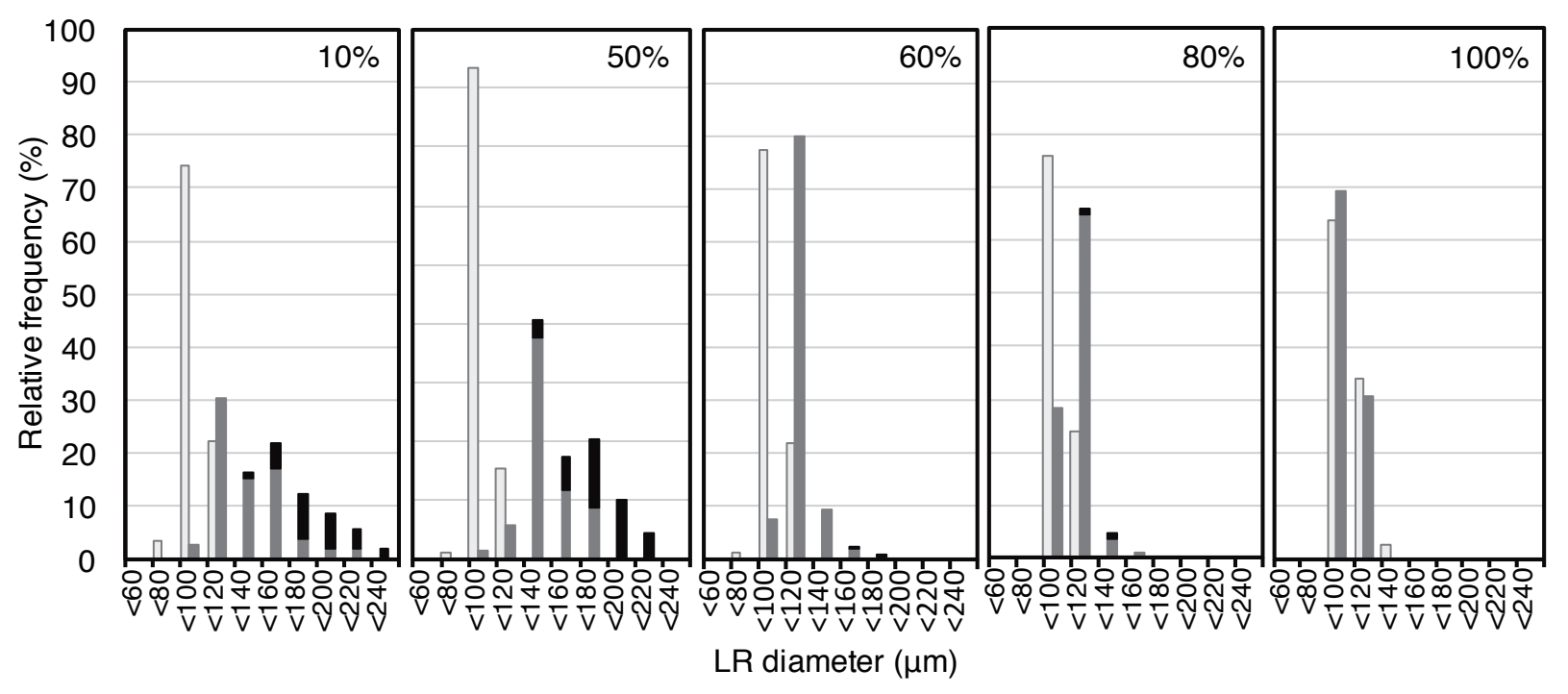

$\square$ - cut -2 nd order LRs $\quad$ + cut -2 nd order LRs $\quad \mathbf{q}+$ cut +2 nd order LRs

Fig. 3. Morphological characteristics of LR that emerged in the different root-cutting ratios. Seminal roots were cut into $10 \%$, $50 \%, 60 \%, 80 \%$, and $100 \%$ root-cutting ratios, and the morphology of the LRs that emerged in the region between excision site and a 10\% higher ratio were analyzed. Morphologies of LRs emerged in the regions corresponding to those of root cuttings in intact seminal roots were analyzed for the control (- cut). (A) Image of a seminal root before the root cutting (bar $=5 \mathrm{~mm}$ ). Arrow heads indicate the cut site of each root-cutting ratio. Squares indicate the regions where morphological characteristics of emerged LRs were measured for each root-cutting ratio. SR, Seminal root; CRs, Crown roots. (B-F) Images of seminal and LRs near the cut site $10 \mathrm{~d}$ after root cutting (bars $=5 \mathrm{~mm}$ ). (G, H) Maximum LR diameter $(\mathrm{G})$ and the number of LRs with second-order LRs $(\mathrm{H})$. Different letters indicate significant differences between groups $(P<0.05)$; values represent means \pm SDs $(\mathrm{n}=10$ plants for each root-cutting ratio). (I) Distributions of LR diameters with or without second-order LRs. 
cutting ratios (Fig. 4A; see Materials and Methods). Anatomical characteristics were virtually identical in the $10 \%$ and $50 \%$ root-cutting ratios (Fig. 4B-D). In the $60 \%$ root-cutting ratios, stele diameter, ground-tissue thickness and the number of groundtissue layers were smaller than those of the $10 \%$ and $50 \%$ root-cutting ratios, but still significantly larger than those of LRs emerged in intact seminal roots. In the $80 \%$ root-cutting ratio, stele diameter of LRs was significantly larger than that of control, whereas ground-tissue thickness and the number of groundtissue layers were not.

Relationship between morphological changes in $L R$ induced by root-tip excision and distance from cut site to each $L R$

We examined how far the effects of the root-tip excision extended in the remaining proximal portions of the $10 \%$ root-cutting ratio by analyzing LR morphology through the $10-60 \%$ ratios from root tip, in which LRP had the same potential to respond to the root-tip excision as shown in Fig. 2B (Fig. 5A; see Materials and Methods). Maximum LR diameter increased in distances up to $15-20 \mathrm{~mm}$ from the cut site (Fig. 5B), whereas significant increases in the maximum length of the first-order LR and emergence of LRs with second-order LRs were limited to distances of $0-5 \mathrm{~mm}$ from the cut site (Fig. 5C, D). The degree of change weakened with distance from the cut site for all LR morphological characteristics (Fig. 5B-D). We next analyzed the relationships between LR morphological characteristics using the three individuals having different distributions of LR diameters and lengths of first-order LRs (Fig. 5E). In the region where both diameter and length of first-order LRs were increased to some extent $(0-10$ $\mathrm{mm}$ from the cut site; Fig. 5B, C), larger diameter LRs tended to become longer and produced secondorder LRs more frequently regardless of distance from the cut site to the LRP in all individuals.
A

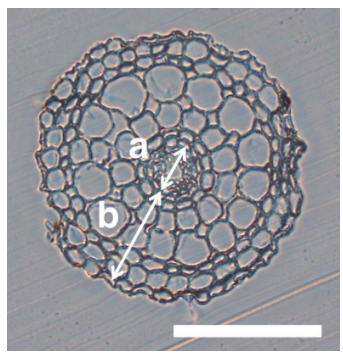

C

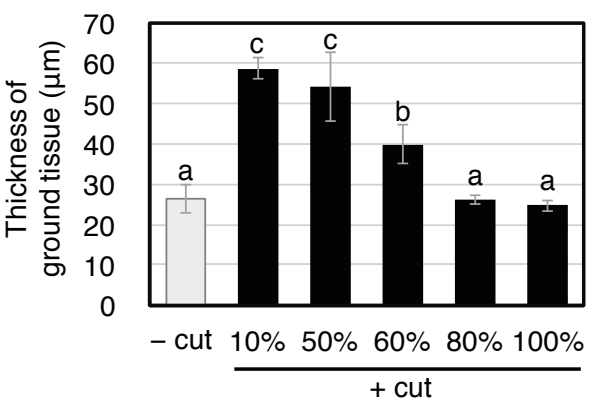

Root-cutting ratio
B

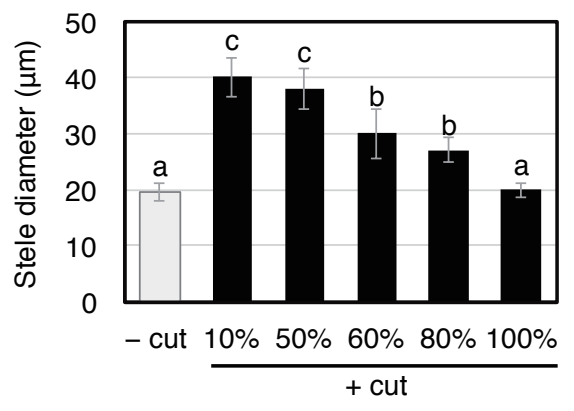

Root-cutting ratio

D

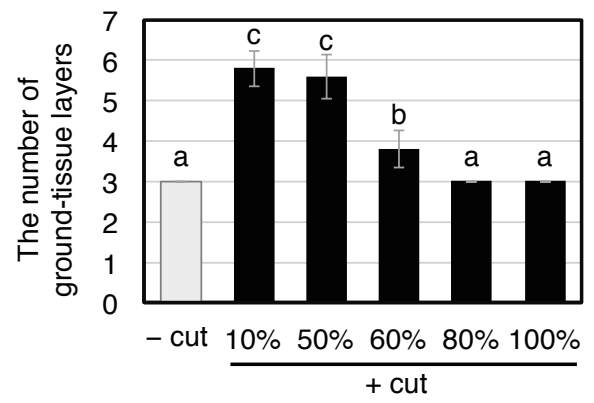

Root-cutting ratio

Fig. 4. Relationship between developmental stage of LRP and anatomical changes of LR induced by seminal-root cutting. The thickest LR among the LRs emerged in each root-cutting ratio were cross-sectioned and their anatomy analyzed. Anatomical characteristics of LRs emerged in the regions corresponding to those of root cuttings in intact seminal roots were analyzed for the control (- cut). (A) Image of a cross-section of LR indicating stele (a) and ground tissue (b) (bar = $100 \mu \mathrm{m}$ ). (B-D) Stele diameter (B), thickness of ground tissue (C), and the number of ground-tissue layers (D) of LRs emerged in different root-cutting ratios. Different letters indicate significant differences between groups $(P<0.05)$; values represent means \pm SDs ( $\mathrm{n}=5$ plants for each root-cutting ratio). 
A

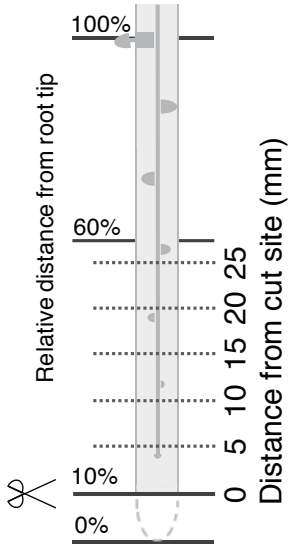

C

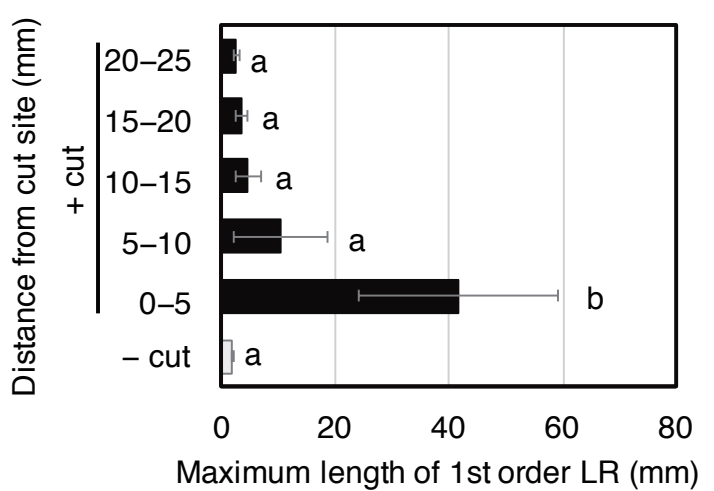

B

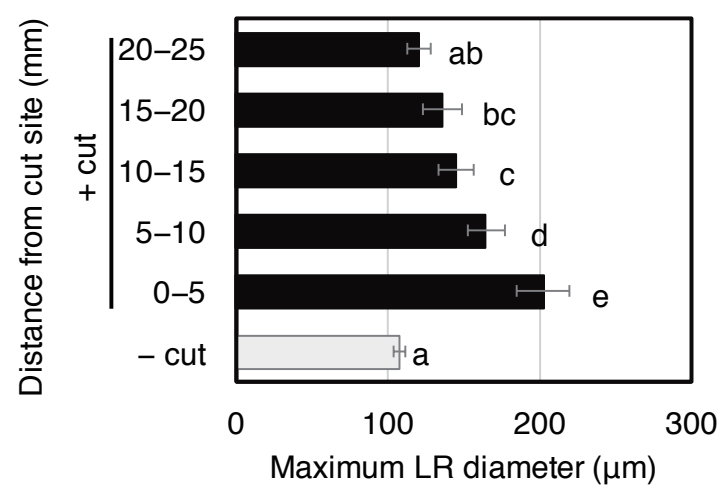

D

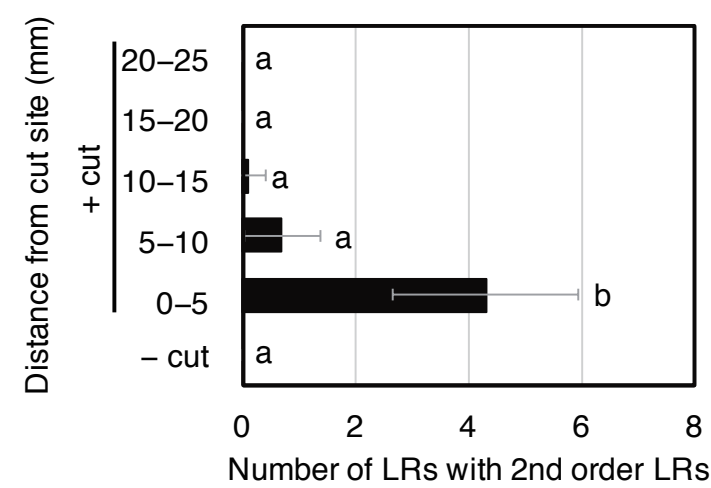

E

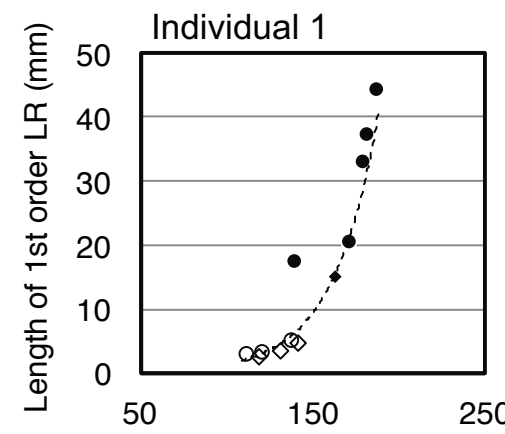

Individual 2

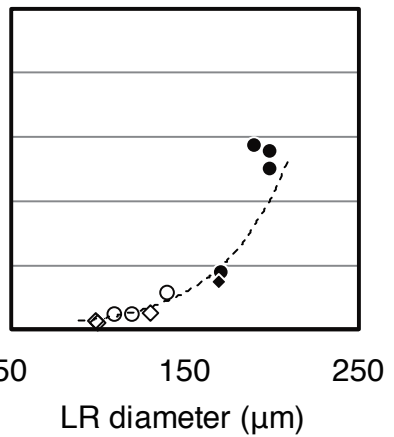

Individual 3

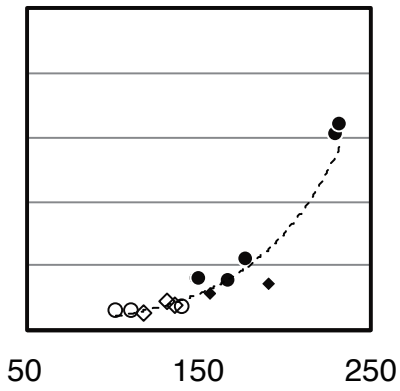

O 0-5 mm - 2nd order LRs

0-5 mm + 2nd order LRs

$\diamond 5-10 \mathrm{~mm}-2$ nd order LRs

Fig. 5. Relationship between distance from the cut site and LR morphology. LRs in the $10 \%$ root-cutting ratio were analyzed every $5 \mathrm{~mm}$ up to a distance of $25 \mathrm{~mm}$ from the cut site. Morphologies of LRs emerged in the regions corresponding to those of the root cutting in intact seminal roots were analyzed for the control (- cut). (A) Schematic diagram showing the region analyzed. (B-D) Means of maximum LR diameter (B), maximum length of first-order LR (C), and the number of LRs with second-order LRs (D) of each region. Different letters indicate significant differences between groups $(P<0.05)$; values represent means \pm SDs $(n=10$ plants). (E) Relationships between LR diameter, length of first-order LR, and production of second-order LRs at distances of $0-5 \mathrm{~mm}$ and 5-10 $\mathrm{mm}$ from the cut site. Data from three different individuals, which have different distributions of LR diameters and lengths of first-order LRs, were shown. 


\section{Discussion}

The morphology of LRs that emerged following the root-tip excision varied continuously depending on the LRP developmental stage at the site of root cutting (Fig. 6A). Relationship between LRP developmental stage and relative distance from root tip to LRP was revealed (Fig. 1C, D), which is consistent with observations for crown roots (Sasaki et al. 1984), and enabled us to examine the relationship between LRP developmental stage and responsiveness to seminal-root cutting by using the various root-cutting ratios. In less than $60 \%$ rootcutting ratio, LR diameter increased to over $160 \mu \mathrm{m}$, with the thick LRs producing second-order LRs (Fig. 2B, Fig. 3B, C, G, H, Fig. 6A). The LRP at the $60 \%$ ratio from root tip had a diameter of $80 \mu \mathrm{m}$ and a length of $70 \mu \mathrm{m}$ (Fig. 1C, D), demonstrating that the diameters of LRP at early developmental stages increase markedly and produce second-order LRs in response to the root-tip excision. Moreover, increases in LR diameter gradually declined with increasing root-cutting ratio, and few LRs produced second-order LRs in more than $60 \%$ root-cutting ratio (Fig. 2B, Fig. 3D-H, Fig. 6A). In addition, small increases in LR diameters were also induced in LRP at later developmental stages. We found that there was a relationship between LRP developmental stage and emergence of LRs with higher order LRs in seminal roots, which is consistent with observation for crown roots (Sasaki et al. 1984), and furthermore, that the diameter of LRs that emerged after the root-tip excision varies continuously depending on the LRP developmental stage at the site of the root cutting.

Seminal-root cutting induces the anatomical changes in LRs and the anatomical characteristics of LRs are different depending on the developmental stage of LRP at the root-cutting site. LRP at early developmental stage markedly increased both the number of ground-tissue layers (and hence increase in thickness of ground tissue) and stele diameter responding to seminal-root cutting (Fig. 4B-D). The increase in the number of ground-tissue layers was driven by the activation of periclinal division of the initial cells in the LRP (Kawata and Shibayama 1965); it may be that seminal-root cutting activates periclinal division of the initial cell in LRP. In later LRP developmental stage, the increase in groundtissue layers decreased as proceeding of LRP development, however, stele diameter was still increased to some extent even in LRP at $80 \%$ ratio from root tip (Fig. 4B, D). Few LRs produced second-order LRs for root-cutting ratios of more than $60 \%$, but LRs with comparatively small diameters at times produced second-order LRs in the $80 \%$ root-cutting ratio (Fig. 3I). Thus, the increase in LR diameter induced by the increase in the number of ground-tissue layers is not necessary for production of second-order LRs, but developed stele may play an important role in the production of higher order LRs. This hypothesis is supported by the finding that thicker roots with second-order LRs tend to have larger stele with thicker late metaxylem, and greater abundances of proto-xylem and protophloem (Sasaki et al. 1981).

The degree of change in LR morphology induced by the root-tip excision is dependent on the distance from the cut site (Fig. 6B). Maximum diameter and length of first-order LRs were relatively larger, and LRs with second-order LRs emerged more frequently the closer to the cut site (Fig. 5B-D, Fig. 6B). The region where increase in LR diameter occurred was further distant from the cut site than those of increase in length of first-order LRs and production of second-order LRs.

Relative LR diameter among the LRs that emerged in the region where both diameter and length of first-order LRs were increased to some extent by the root-tip excision determines the length of the first-order LRs and whether or not the LR produces second-order LRs (Fig. 6B). Relatively large diameter LRs were longer than smallerdiameter LRs and produced second-order LRs in the $10 \%$ root-cutting ratio regardless of distance from the cut site (Fig. 5E, Fig. 6B), indicating that relative LR diameter among the LRs that emerged from the same parent root after the root-tip excision might determine the length of the first-order LR and whether the LR produces second-order LRs. Root apical diameter was positively correlated with root elongation rate (Cahn et al. 1989, Pages 1995, Lecompte and Pages 2006, Wu et al. 2016), and is regarded as an indicator of the sink strength of a root (Thaler and Pages 1999). Thus, increased assimilate supply to first-order LRs of larger diameter and sink strength as a result of excision of a seminal root tip induces preferential elongation and production of second-order LRs. Based on these findings, we are now planning to undertake several experiments to further our understanding of the molecular mechanisms underlying compensatory LR growth.

\section{Acknowledgements}

We thank Ms. Kimiyo Inukai, Ms. Eiko Murakami, and Ms. Saki Nishiuchi for their valuable technical supports. This work was supported by PRESTO JST, the Grant-in-Aid from the Japan Society for the Promotion of Science (No. 15H04435), and JST/JICA, 

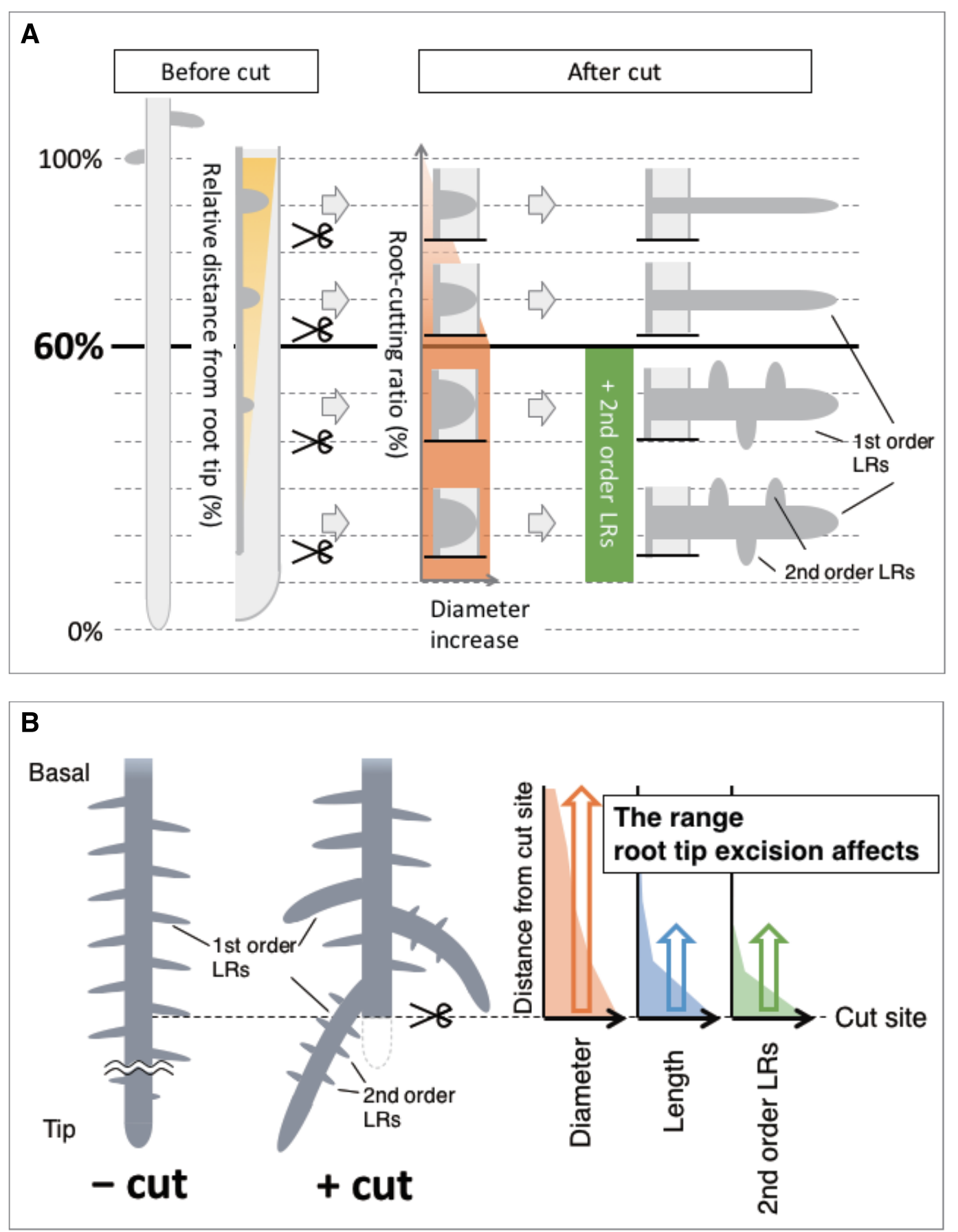

Fig. 6. (A) Schematic model illustrating the relationship between the morphological changes in LRs induced by the root-tip excision and LRP developmental stage. (Left) Prior to root cutting, the LRP developmental stage proceeded along the seminal root, which positively correlated with relative distance from root tip within root tip to the LR emergence site. (Middle) LRP and LR diameters increased as a result of root cutting, with the degree of change in diameter depending on the LRP developmental stage at the site of the root cutting. For root-cutting ratios of less than $60 \%$, diameters increased by more than $160 \mu \mathrm{m}$, but gradually declined for root-cutting ratios of more than $60 \%$. (Right) Large-diameter LRs often produced second-order LRs in the region for root-cutting ratios of less than $60 \%$, but second-order LRs rarely emerged from first-order LRs for root-cutting ratios of more than $60 \%$. (B) Schematic model showing the relationship between morphological changes in LR and distance from the cut site. The region where increase in LR diameter occurred was further distant from the cut site than those of increases in length of first-order LR and production of second-order LRs. Thicker LRs grew longer and produced second-order LRs at a greater frequency regardless of distance from root tip. 
the Science and Technology Research Partnership for Sustainable Development (SATREPS).

\section{References}

Atwell BJ 1990 The effect of soil compaction on wheat during early tillering. I. Growth, development and root structure. New Phytol. 115: 29-35.

Bingham IJ, Bengough AG 2003 Morphological plasticity of wheat and barley roots in response to spatial variation in soil strength. Plant Soil 250: 273-282.

Cahn MD, Zobel RW, Bouldin DR 1989 Relationship between root elongation rate and diameter and duration of growth of lateral roots of maize. Plant Soil 119: 271-279.

Fox J 2005 The R commander: a basic-statistics graphical user interface to R. J. Stat. Softw. 14: 1-42.

Goss MJ 1977 Effects of mechanical impedance on root growth in barley (Hordeum vulgare L.). I. Effects on the elongation and branching of seminal root axes. J. Exp. Bot. 28: 96-111.

Haling RE, Simpson RJ, Culvenor RA, Lambers H, Richardson AE 2011 Effect of soil acidity, soil strength and macropores on root growth and morphology of perennial grass species differing in acid-soil resistance. Plant, Cell Environ. 34: 444-456.

Hasegawa J, Sakamoto Y, Nakagami S, Aida M, Sawa S, Matsunaga S 2016 Three-dimensional imaging of plant organs using a simple and rapid transparency technique. Plant Cell Physiol. 57: 462-472.

Kawata S, Shibayama B 1965 On the lateral root primordia formation in the crown roots of rice plant. Proc. Crop Sci. Soc. Japan 33: 423-431.

Kono Y, Igeta M, Yamada N 1972 Studies on the developmental physiology of the lateral roots in rice seminal roots. Proc. Crop Sci. Soc. Japan 41: 192-204.

Lecompte F, Pagès L 2006 Apical diameter and branching density affect lateral root elongation rates in banana. Environ. Exp. Bot. 59: 243-251.

Montagu KD, Conroy JP, Atwell BJ 2001 The position of localized soil compaction determines root and subsequent shoot growth responses. J. Exp. Bot. 52: 2127-2133.

Niones JM, Inukai Y, Suralta RR, Yamauchi A 2015 QTL associated with lateral root plasticity in response to soil moisture fluctuation stress in rice. Plant Soil 391: 63-75.

Pagès L 1995 Growth patterns of the lateral roots of young oak (Quercus robur) tree seedlings. Relationship with apical diameter. New Phytol. 130: 503-509.

Sasaki O, Yamazaki K, Kawata S 1981 The relationship between the diameters and the structures of lateral roots in rice plants. Jpn. J. Crop Sci. 50: 476-480.

Sasaki O, Yamazaki K, Kawata S 1984 The development of lateral root primordia in rice plants. Jpn. J. Crop Sci. 53: 169-175.
Shierlaw J, Alston AM 1984 Effect of soil compaction on root-growth and uptake of phosphorus. Plant Soil 77: 1528.

Thaler P, Pagès L 1999 Why are laterals less affected than main axes by homogeneous unfavourable physical conditions? A model-based hypothesis. Plant Soil 217: 151-157.

Wang H, Inukai Y, Yamauchi A 2006 Root development and nutrient uptake. CRC Crit. Rev. Plant Sci. 25: 279-301.

White RG, Kirkegaard JA 2010 The distribution and abundance of wheat roots in a dense, structured subsoil implications for water uptake. Plant, Cell Environ. 33: 133-148.

Wu Q, Pagès L, Wu J 2016 Relationships between root diameter, root length and root branching along lateral roots in adult, field-grown maize. Ann. Bot. 117: 379-390.

Yamauchi A, Kono Y, Tatsumi J 1987 Quantitative analysis on root system structures of upland rice and maize. Jpn. J. Crop Sci. 56: 608-617.

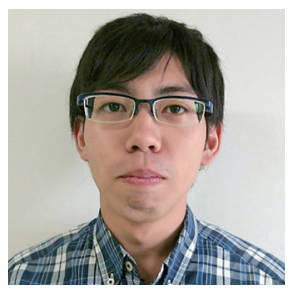

Tsubasa Kawai is a Master's course student and trying to reveal the molecular mechanisms underlying lateral root development in rice.

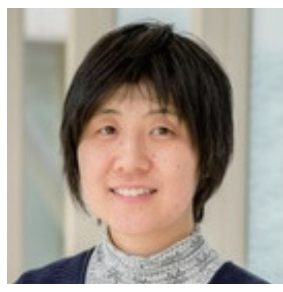

Dr. Misuzu Nosaka-Takahashi is researching on rice development and epigenetic regulation.

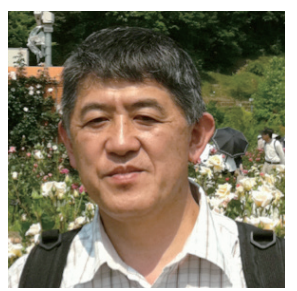

Dr. Akira Yamauchi has been studying the functional roles of root system in crop production especially under unfavorable conditions such as water stress, nutrient deficiency and the identification of root ideotype under each environment.

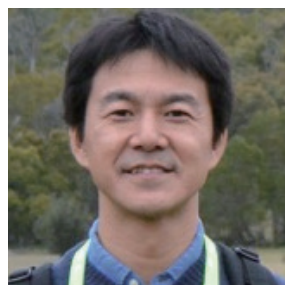

Dr. Yoshiaki Inukai tries to improve rice root system architecture through understanding of genetic mechanism regulating root formation in rice. 